\title{
FIVE MASS POWER TRANSMISSION LINE OF A SHIP COMPUTER MODELLING*
}

\author{
Alexander Borisoff Kazakoff, Boycho Ivanov Marinov \\ Institute of Mechanics, Bulgarian Academy of Science, \\ Acad. G. Bonchev St., Bl. 4, 1113 Sofia, Bulgaria, \\ e-mails: alex_kazakoff@yahoo.co.uk, boicho_marinoff@yahoo.co.uk, \\ boicho_marinoff@abv.bg
}

[Received 28 October 2015. Accepted 29 February 2016]

\begin{abstract}
The work, presented in this paper, appears to be a natural continuation of the work presented and reported before, on the design of power transmission line of a ship, but with different multi-mass model. Some data from the previous investigations are used as a reference data, mainly from the analytical investigations, for the developed in the previous study, frequency and modal analysis of a five mass model of a power transmission line of a ship. In the paper, a profound dynamic analysis of a concrete five mass dynamic model of the power transmission line of a ship is performed using Finite Element Analysis (FEA), based on the previously recommended model, investigated in the previous research and reported before. Thus, the partially validated by frequency analysis five mass model of a power transmission line of a ship is subjected to dynamic analysis. The objective of the work presented in this paper is dynamic modelling of a five mass transmission line of a ship, partial validation of the model and von Mises stress analysis calculation with the help of Finite Element Analysis (FEA) and comparison of the derived results with the analytically calculated values. The partially validated five mass power transmission line of a ship can be used for definition of many dynamic parameters, particularly amplitude of displacement, velocity and acceleration, respectively in time and frequency domain. The frequency behaviour of the model parameters is investigated in frequency domain and it corresponds to the predicted one.

KEY WORDS: Modal and frequency analysis, power transmission line of a ship, finite element analysis (FEA), five mass dynamic model, natural frequencies, dynamic simulation, dynamic analysis.
\end{abstract}

\footnotetext{
${ }^{*}$ Corresponding author e-mail: alex_kazakoff@yahoo.co.uk
} 


\section{Introduction}

The power transmission lines of ships are complex mechanical systems $[1,2]$. They serve to deliver the working torque from the engine to the marine propeller $[4,5,10,11]$. Every transmission line is composed of a crank shaft, a flywheel, one or more intermediate shafts, a support shaft and a marine propeller $[4,5]$. This complex dynamic system is subjected to variable moments, which excite torsion vibrations $[4,5,6,7,8,9]$. Dynamic model of a five mass power transmission line of a ship - basic configuration is presented in Fig. 1 [12].

In this study, the first part of the investigation, the most suitable, validated and reliable model for the dynamic analysis is to be chosen, performed in [12]. The second part of the study, which is a subject of this investigation, is the dynamic analysis, when all the model shafts are going to be loaded with the real external torques, applied on the four discs and on the propeller, respectively.

Two basic conclusions could be used, derived in [12], analysing the results from the analytical and FEA performed in the study:

- There is a relatively acceptable confirmation between the analytical results, used as a basis in this investigation and the results followed by the FEA. The results derived by the computer analysis are with damping that is why they are expected to be with acceptable precision [12].

- As a most reliable and precise model, as compared to the analytical analysis, selected for the future dynamic analysis is Model 3, built up and shown in the study [12]. This model is presented in Fig. 2 in the present work.

The objective of the work presented in this paper is dynamic modelling of a five mass model transmission line of a ship, partially validation of the model in the previous study [12], by modal and frequency analysis, and calculating von Mises stress with the help of Finite Element Analysis (FEA) and comparison with the analytically calculated values. The partially validated five mass power transmission line of a ship model can be used for definition of many dynamic parameters, particularly amplitude of displacement, velocity and acceleration in time and frequency domain. The frequency behaviour of model parameters is investigated in frequency domain and it corresponds to the predicted one.

\section{Mechanical modelling}

The selected Model 3 [12] will be used further on, in the planned future dynamic analysis of a five mass power transmission line of a ship, in order to define the shear stresses, typical for the axle pure torsion, as a characteristic loading of the system [12]. 


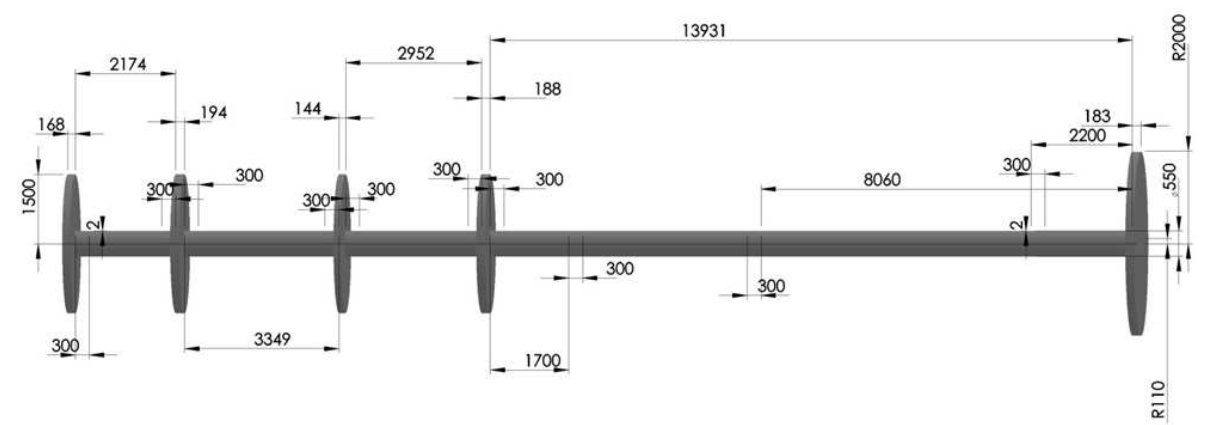

Fig. 1. Free-Free suspension of the five mass model of a power transmission line of a ship - Model 1 - basic geometry dimensions in [mm]



Fig. 2. The five mass model suspension with bearings in the middle between the discs - Model 3- basic suspension dimensions in [mm]

After thorough consideration of the investigated models, three of which are given in [12], also analysing the results, we strongly recommend the model - Model 3, shown in Fig. 4 [12], and presented here in Fig. 2 below, to be selected as a representative for further investigation in the dynamic analysis, which is going to be shown in this article.

Input loading torques, starting from left to right (see Fig. 2), are $M_{1}$, $M_{2}, M_{3}$ and $M_{4}$ (defined below in Fig. 3), acting on the corresponding discs and the resistance moment $M_{R}$, acting on the propeller, in a reverse direction with respect to the previous moments. These moments are presented in Fig 3. They can be defined by the following equations:

$$
\begin{aligned}
& M_{1}=2 M_{m}+M_{k} \sin \left(3 \omega t+\delta_{13}\right)+M_{k} \sin \left(3 \omega t+\delta_{23}\right), \\
& M_{2}=2 M_{m}+M_{k} \sin \left(3 \omega t+\delta_{33}\right)+M_{k} \sin \left(3 \omega t+\delta_{43}\right), \\
& M_{3}=2 M_{m}+M_{k} \sin \left(3 \omega t+\delta_{53}\right)+M_{k} \sin \left(3 \omega t+\delta_{63}\right), \\
& M_{4}=M_{m}+M_{k} \sin \left(3 \omega t+\delta_{73}\right), \\
& M_{R}=3.5 e+5,
\end{aligned}
$$

where $M_{m}=0.5 e+5[\mathrm{Nm}]$ is the average torque of the torques $M(t)$ decomposition of the gas and the inertia forces, as well as the force-loads acting on each 

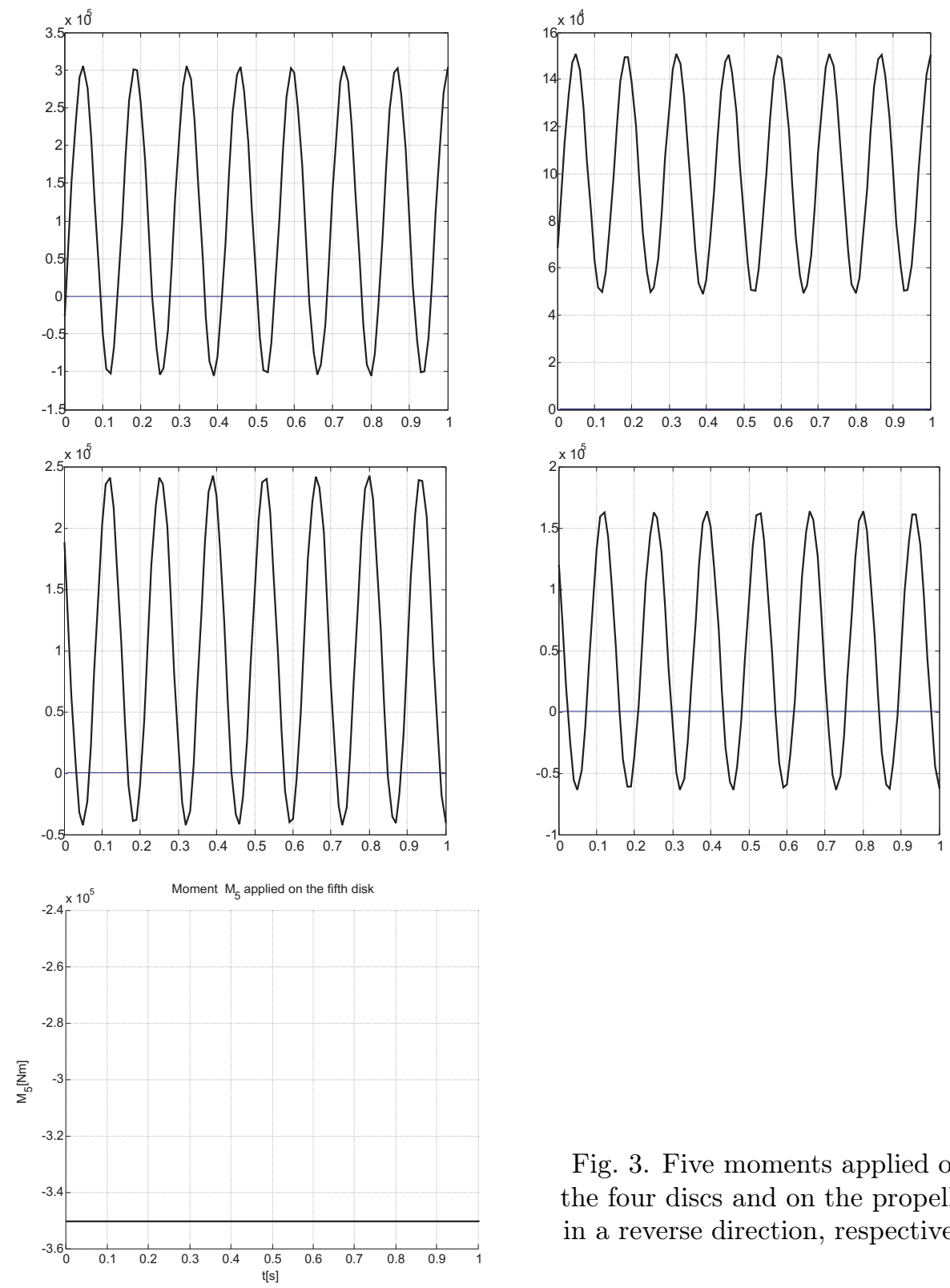

Fig. 3. Five moments applied on the four discs and on the propeller in a reverse direction, respectively

piston, $M_{k}=1.14 e+5[\mathrm{Nm}]$ is the amplitude of the harmonic components of the torques, excited from the $k$-th harmonic [4]. In this case, the resonance is predetermined from the third harmonic, i. e. $k=3$. The resistant torque is $M_{R}=3.5 e+5[\mathrm{Nm}]$, excited by the propeller [4]. We assume that it is equal to 
a constant. Crankshaft angular velocity is $\omega=12.92\left[\mathrm{~s}^{-1}\right]$. Phase angles of the third harmonic $(k=3)$ of the torques, acting on the $i$-th and the first crank are:

$$
\begin{gathered}
\delta_{13}=-0.2093,[4], \delta_{73}=2.4821, \delta_{23}=5.1733, \delta_{53}=7.8646, \delta_{43}=10.5559, \\
\delta_{33}=13.2472, \delta_{63}=15.9385 .
\end{gathered}
$$

Order of cylinder ignition is 1-7-2-5-4-3-6. The time variation for the numerical calculations of the torques $M_{1}, M_{2}, M_{3}$ and $M_{4}$ is $t=0: 0.01: 1$. The graphical representation of the torques in consideration are represented below, starting from left to right, considering the dynamic model, presented in Fig. 2.

\section{Analytical and numerical results}

3.1. Theoretical calculations for determining the torsional stresses in all sections of the dynamic model, caused by resonance mode

The five mass model natural frequencies were calculated in previous reports $[1,3]$. The first natural frequency is $\omega_{1}=38.77\left[\mathrm{~s}^{-1}\right]$. If the crankshaft rotates with angular velocity $\omega=12.92\left[s^{-1}\right]$, the third vibration harmonic will excite vibrations with resonance amplitude. Complete tangential (torsional) shear stresses in this case can be calculated by the following formula [4, 5]:

$$
\tau_{i, i+1}=\tau_{i, i+1}^{s t}+\tau_{i, i+1}^{d y n}, \quad(i=1 \div 4),
$$

where $\tau_{i, i+1}^{s t}$ and $\tau_{i, i+1}^{d y n}$ are the static and the dynamic components of the calculated stresses. Static components can be calculated from the known in the literature $[4,5,16]$ formula, expressed as:

$$
\tau_{i, i+1}^{s t}=\frac{M_{m i}}{W_{i, i+1}}, \quad(i=1 \div 4),
$$

where $M_{m i}$ are the average moments, acting on each section, as: $M_{m 1}=1 e+5$ $[\mathrm{Nm}], M_{m 2}=2 e+5[\mathrm{Nm}], M_{m 3}=3 e+5[\mathrm{Nm}], M_{m 4}=3.5 e+5[\mathrm{Nm}] . W_{i, i+1}$ is a resistance (elastic section modulus) moment.

$$
W_{i, i+1}=\frac{\pi D_{i, i+1}^{3}}{16}\left(1-\alpha^{4}\right), \quad \alpha=\frac{d_{i, i+1}}{D_{i, i+1}}
$$

where $d_{i, i+1}$ and $D_{i, i+1}$ are the internal and the external diameters of the separate shafts. The ratio $\alpha=0,4$. was established by constructive and design 
considerations. These moments have the same values $W_{i, i+1}=0.0318\left[\mathrm{~m}^{3}\right]$, because the shaft diameters at the corresponding sections possess the same values $D_{i, i+1}=0.55[\mathrm{~m}]$. Dynamic components can be calculated by the following expressions $[4,5]$ :

$$
\tau_{i, i+1 d y n}=\frac{c_{i, i+1}\left(\alpha_{i 1}-\alpha_{i+1,1}\right)}{W_{i, i+1}} \Phi_{13}
$$

where: $\Phi_{13}$ is the resonant amplitude of the first disc, caused by the third harmonic of the first natural frequency. For its determination, numerical results are used, obtained in previous work $[1,3]$. In the particular case $\Phi_{13}=0.00409$ [rad]. The mode shapes for the first natural frequency $\omega_{1}$ have been determined, using the following expressions:

$$
\begin{aligned}
& \alpha_{11}=1, \\
& \alpha_{21}=1-\frac{I_{1}^{*} \omega_{1}^{2}}{c_{12}^{*}} \\
& \alpha_{31}=\alpha_{2 j}-\frac{\omega_{1}^{2}}{c_{23}^{*}}\left(I_{1}^{*}+I_{2}^{*} \alpha_{21}\right), \\
& \alpha_{41}=\alpha_{31}-\frac{\omega_{1}^{2}}{c_{34}^{*}}\left(I_{1}^{*}+I_{2}^{*} \alpha_{21}+I_{3}^{*} \alpha_{31}\right), \\
& \alpha_{51}=\alpha_{41}-\frac{\omega_{1}^{2}}{c_{45}^{*}}\left(I_{1}^{*}+I_{2}^{*} \alpha_{21}+I_{3}^{*} \alpha_{31}+I_{4}^{*} \alpha_{41}\right) .
\end{aligned}
$$

The mass moments of inertia $I_{i}^{*}$ and the dynamic model elastic torque constants $c_{i, i+1}^{*}, \quad(i=1 \div 4)$ are determined in previous works $[1,3,12]$. Their values and values of their natural frequencies and forms are mapped in Table 1.

Table 1. Values of the mass moments of inertia and values of their natural frequencies and forms

\begin{tabular}{|l|c|c|c|c|c|}
\hline$I_{i}^{*}(i=1 \div 5)\left[\mathrm{kg} \mathrm{m}^{2}\right]$ & 10500 & 12110 & 9010 & 11710 & 36080 \\
\hline$c_{i, i+1}^{*},(i=1 \div 4)[\mathrm{Nm} / \mathrm{rad}]$ & $3.010 \mathrm{e}+8$ & $2.041 \mathrm{e}+8$ & $2.270 \mathrm{e}+8$ & $0.505 \mathrm{e}+8$ & - \\
\hline$\alpha_{i 1},(i=1 \div 5)$ & 1 & 0.9476 & 0.7858 & 0.5934 & -0.4782 \\
\hline
\end{tabular}

The estimated full tangential stresses are presented in the Table 2 .

The calculated tangential tensions are compared with the admissible values. In this case, the admissible (shear stress) tension is defined to be: $[\tau]=235.10^{5} \mathrm{~Pa}$. We estimate that the condition $\tau_{i, i+1} \leq[\tau],(i=1 \div 4)$ is satisfied. The selected diameter, however, can be reduced and adopt the final size of $D=0.52[\mathrm{~m}]$. 
Five Mass Power Transmission Line of a Ship Computer Modelling

Table 2. Tangential tensions (shear stress) in the separate sections

\begin{tabular}{|l|c|c|c|c|}
\hline Tangential tensions & $\tau_{12} \cdot 10^{5}[\mathrm{~Pa}]$ & $\tau_{23.10^{5}[\mathrm{~Pa}]}$ & $\tau_{34} \cdot 10^{5}[\mathrm{~Pa}]$ & $\tau_{45} \cdot 10^{5}[\mathrm{~Pa}]$ \\
\hline Static & 31.44654 & 62.89308 & 94.33962 & 110.06289 \\
\hline Dynamic & 20.28588 & 42.47347 & 56.17293 & 69.60177 \\
\hline Full & 51.73242 & 105.36655 & 150.51255 & 179.66466 \\
\hline
\end{tabular}

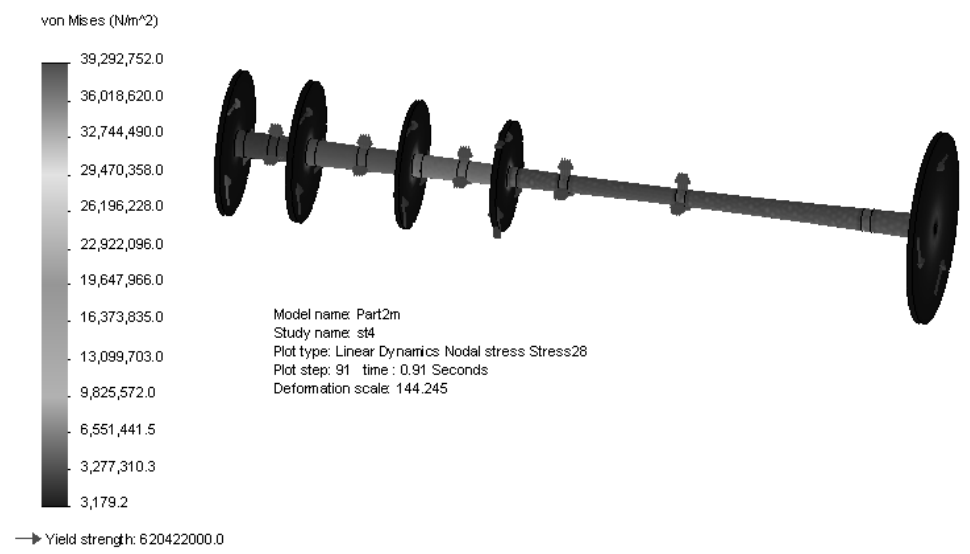

Fig. 4. Von Mises and Yield strength plot of the basic Model 3

\subsection{Numerical results}

The established dynamic model, presented in Fig. 2, was solved numerically and the solution is presented in Fig. 4. It shows the von Mises stress and figs 5-13 show amplitudes of displacement, velocity and acceleration in time domain of a typical node on the periphery of the shaft, near the propeller and their transforms in frequency domain, using Fast Fourier Transformation (FFT) $[13,14,15]$. The figure shows that the von Mises stress distribution in all the shaft sections, correspond to the theoretically calculated one and presented in Table 2. In the numerical calculations, a modal damping of a value of 0.1 is used in the modelling of this highly damped dynamic system. This value is selected after data taken from literature, but a more precisely selected value after a practical experiment, could give even better results.

\section{Comparison of the results and analysis}

The numerical results, presented in Figs 5-13 show a stable dynamic behaviour of the dynamic system, and particularly von Mises stress distribution, amplitude of displacement, velocity and acceleration, respectively of some 


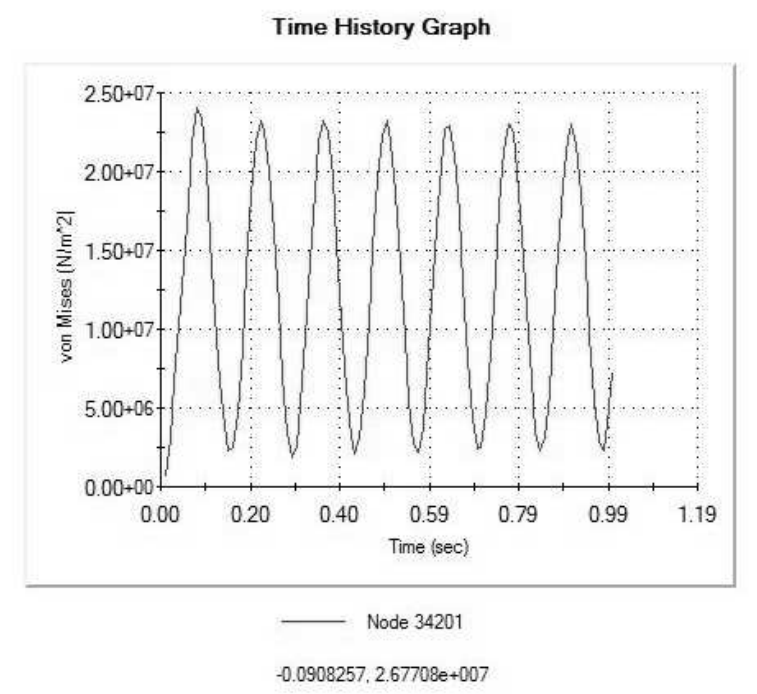

Fig. 5. Von Mises stress on the periphery of the shaft at node 34201 (just before the propeller) - Time History Graph
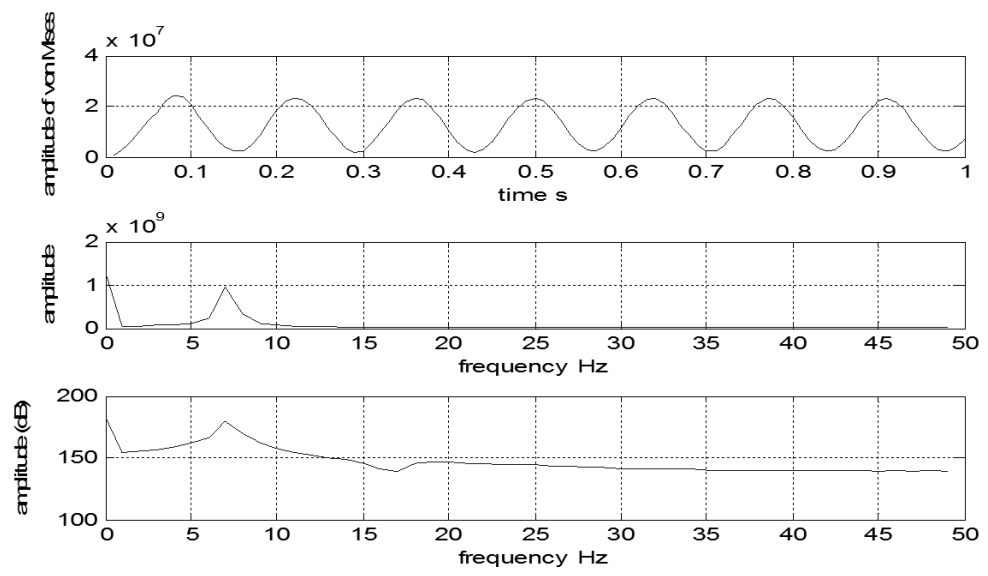

Fig. 6. Von Mises stress on the periphery of the shaft at node 34201 transferred in frequency domain using FFT - resonance at zero and at $7 \mathrm{~Hz}$

typical nodes on the periphery of the shat and tangential to the longitudinal axis. For the sake of illustration, here, one particular node is selected - 34201, allocated on the periphery of the shaft, very close to the propeller, which theoretically should be one of the most loaded positions. 
Five Mass Power Transmission Line of a Ship Computer Modelling

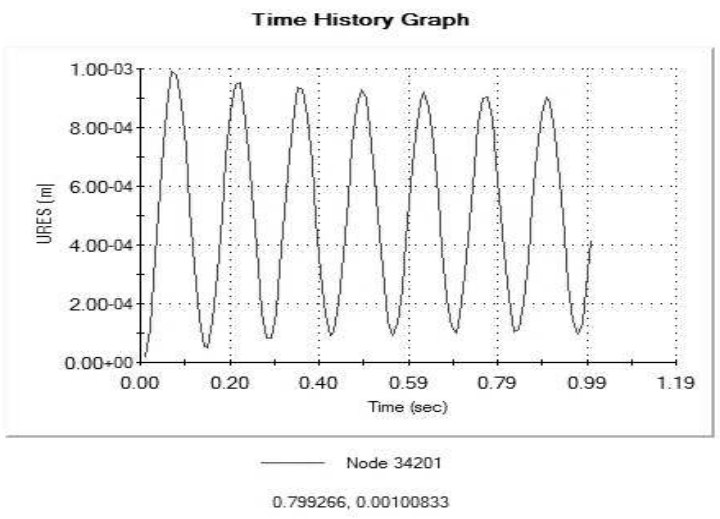

Fig. 7. Displacement (general) at node 34201 on the periphery of the shaft - Time History Graph

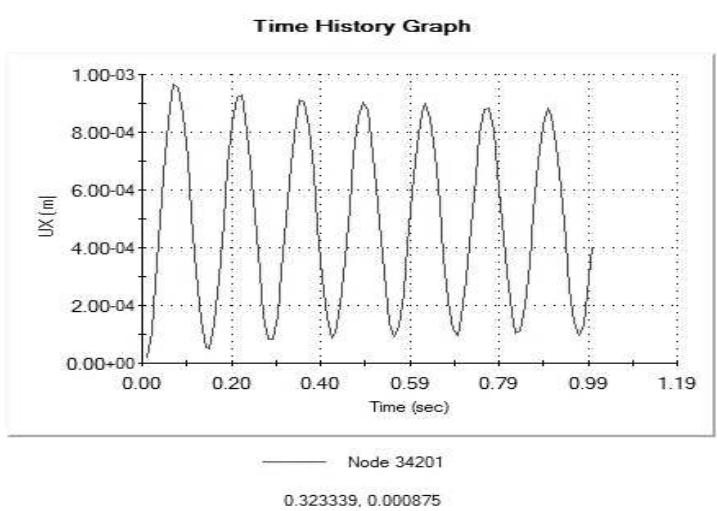

Fig. 8. Displacement (along coordinate x-peripheral to the shaft) at node 34201 on the periphery of the shaft - Time History Graph - axis $\mathrm{x}$

Figure 5 shows the von Mises stress distribution in time domain and after transformation in frequency domain, using Fast Fourier Transformation (FFT) the graphical interpretation is presented in Fig. 6. Resonances are at 0 and at $7 \mathrm{~Hz}$. Solution is stable at the equilibrium.

Figures 7 and 8 show the displacement of the typical node and the resultant displacement, and the displacement along axis $\mathrm{x}$ perpendicular to the longitudinal shaft axis and the node is situated on the surface of the shaft periphery. It is seen, however, that both graphs are almost identical, which 

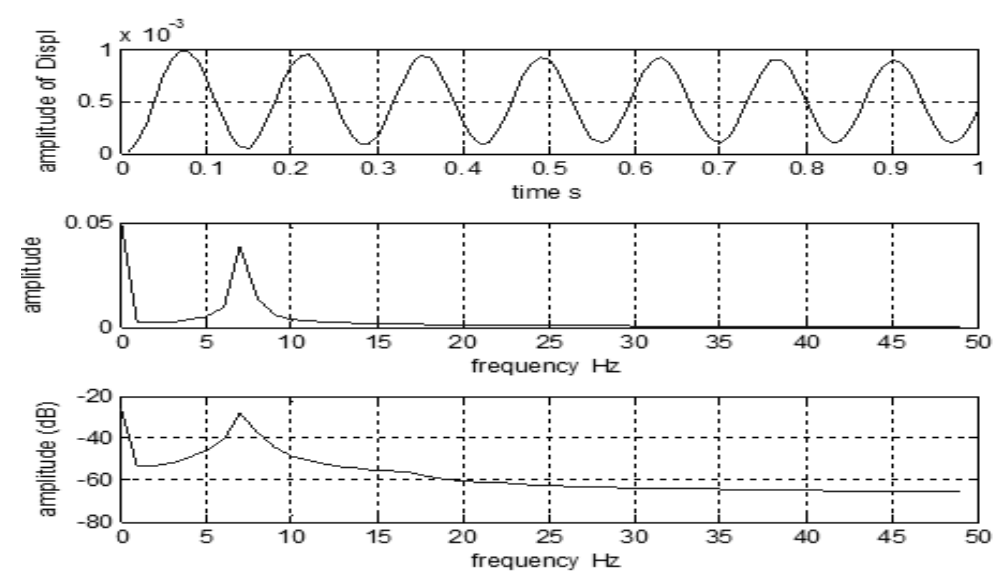

Fig. 9. Displacement at node 34201 on the periphery of the shaft transferred in frequency domain using FFT - resonance at zero and at $7 \mathrm{~Hz}$



Fig. 10. Velocity at node 34201 on the periphery of the shaft (along coordinate $\mathrm{x}$-peripheral to the shaft) - Time History Graph

could be expected, because of the fact that the shaft is subjected to pure torsion and the rotation (i. e. the displacement along axis $\mathrm{x}$ ) is the main system motion. Figure 9 shows the displacement time history graph transformed in frequency domain using FFT. Again, the resonances are at 0 and $7 \mathrm{~Hz}$, which gives a very good agreement with the analytical results.

Figures 10 and 12 show amplitude of velocity and acceleration of the 

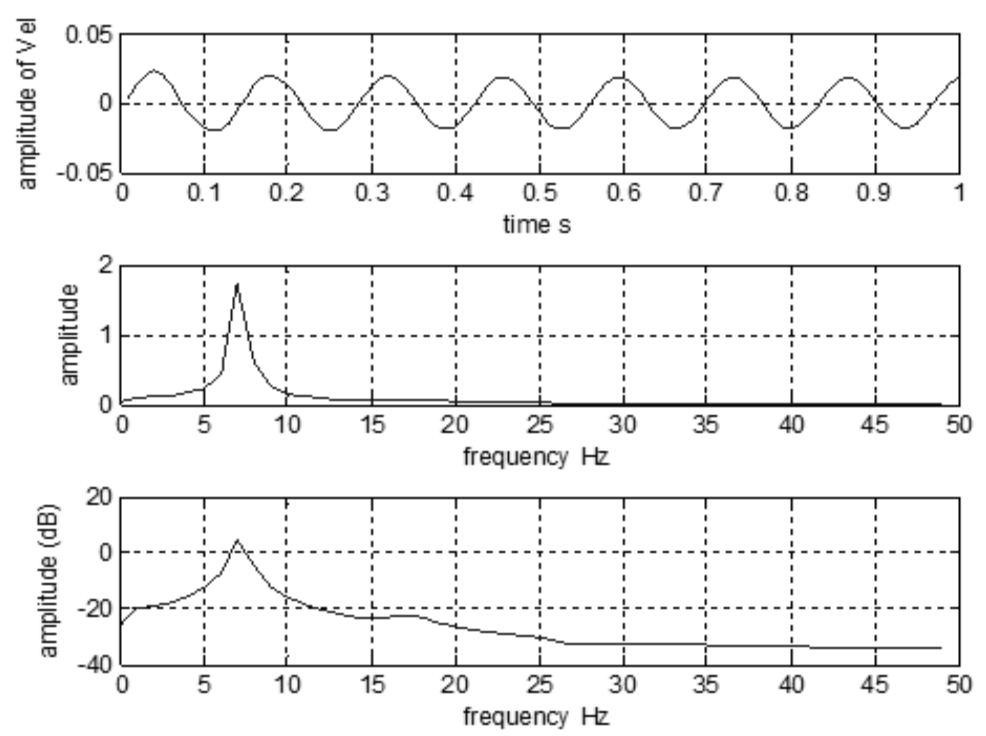

Fig. 11. Velocity at node 34201 on the periphery of the shaft - transferred in frequency domain using FFT - resonance at $7 \mathrm{~Hz}$

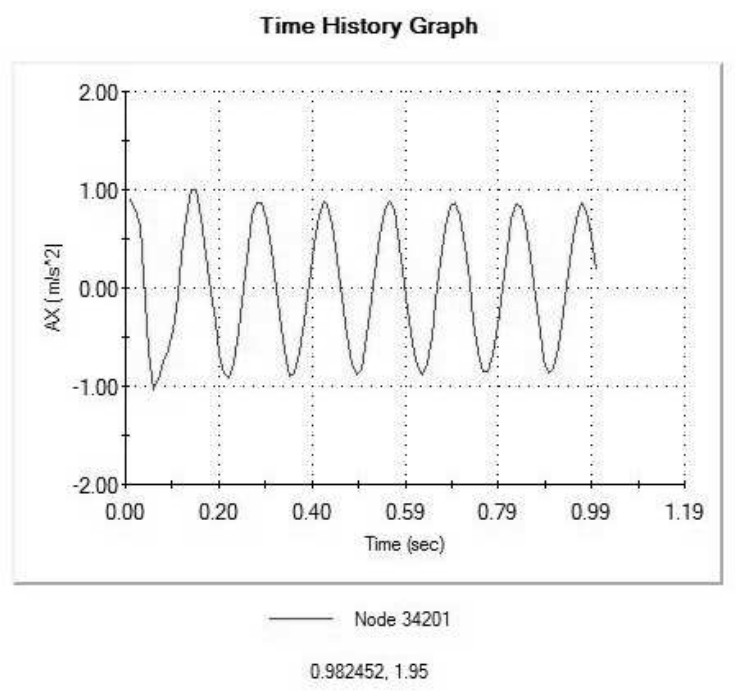

Fig. 12. Acceleration at node 34201 on the periphery of the shaft (along coordinate x-peripheral to the shaft) - Time History Graph 

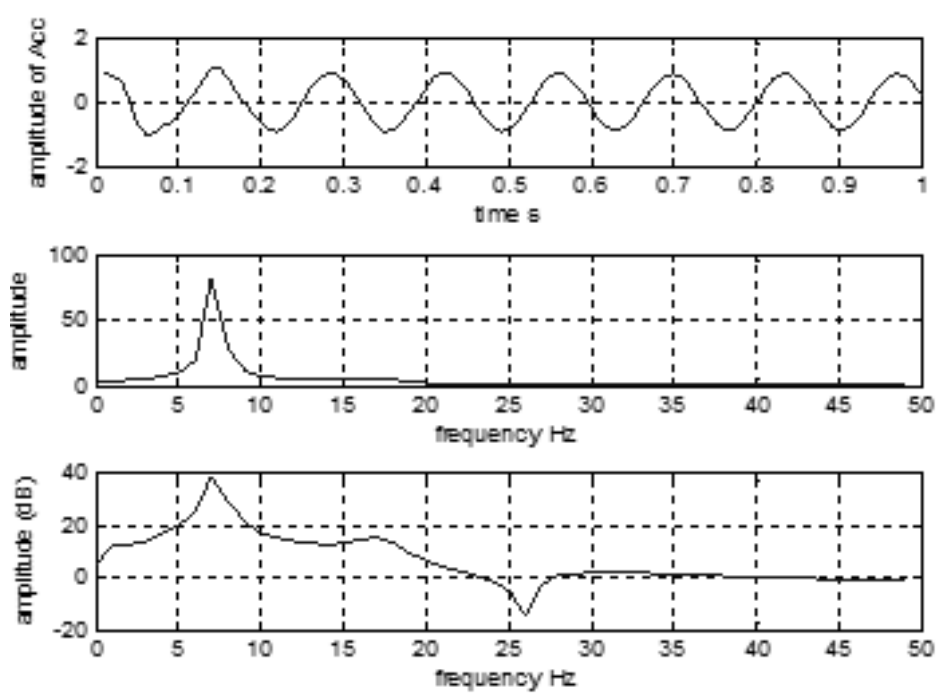

Fig. 13. Acceleration at node 34201 on the periphery of the shaft (along coordinate $\mathrm{x}$-peripheral to the shaft), transferred in frequency domain, using FFT - resonance at $7 \mathrm{~Hz}$
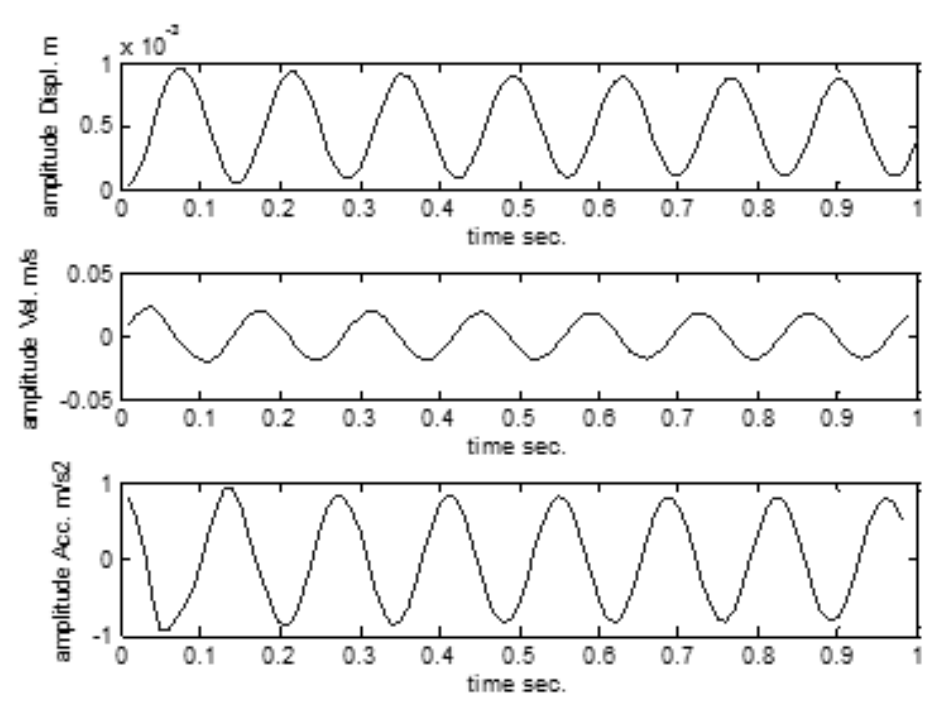

Fig. 14. Double differentiation of displacement, presented in Fig. 8, which gives amplitude of velocity-second plot and amplitude of acceleration-third plot, respectively. Results coincide with those presented in Figs 10 and 12, respectively 
Five Mass Power Transmission Line of a Ship Computer Modelling

typical node in time domain and Figs 11 and 13 show the transformed Figs 10 and 12 in frequency domain, respectively. Resonances could be clearly seen at $7 \mathrm{~Hz}$ in both graphs, which is another confirmation of the previous results and a very good agreement with the analytical ones.

In Fig. 14, a double differentiation of the amplitude of displacement, shown in Fig 8 is presented. This is a kind of an independent check for results' correction. Second plot corresponds to the amplitude of velocity, presented in Fig. 10, and the third plot correspond to the amplitude of acceleration, presented in Fig. 12, as expected.

\section{Conclusions}

In this paper, the partially validated by frequency analysis five mass model of a power transmission line of a ship, reported before, is subjected to dynamic analysis. The predicted numerical results are compared with the analytical ones and the following basic conclusions can be derived:

1. A five mass dynamic multi-mass model is used in the present investigation, partially validated by frequency analysis and reported before.

2. Numerical results fully coincide with the theoretically calculated ones and reported before.

3. Analysis of the results in frequency domain show that the first two system resonances at 0 and $7 \mathrm{~Hz}$ are well defined, and there is a good agreement with the predictions of the deliberately performed frequency analysis, reported before.

4. Presented solutions are stable in the equilibrium.

5. The well defined system stress distribution allows a correct selection of the shaft diameter.

The results, achieved in this study, can be a god application in the ship transmission parameters design and calculation in addition to the analytical investigations, popular so far. The computer modelling allows flexibility in the geometrical and system parameters, which are supposed to meet the needs of every individual case study.

\section{REFERENCES}

[1] Marinov, B., H. Hristov. Links Dimension Optimization in a Three Mass System of Multi Cylinder Internal Combustion Engine. Archives of Transport, 13 (2001), No. 1, 39-52. 
[2] Marinov, B., H. Hristov, G. Vukov. Link Dimension Approach to a Two Mass System of Multi Cylinder Internal Combustion Engine. Mechanics of Machines, 28 (1999), No. 4, 50-55 (In Bulgarian).

[3] Marinov, B. On the Design of Power Transmission Line of a Ship. Archives of Transport, 14 (2002), No. 4, 53-70.

[4] Minchev, N. Dynamics of Ship Machine, Sofia, Military Press, 1983 (in Bulgarian).

[5] Minchev, N., H. R. Draganchev. Strengths and Vibrations of Ship Machinery, Sofia, Galaktika Press, 1992 (In Bulgarian).

[6] Zlatanov, Z. T. Stress and Strain Definition of an Open Profile Thin-walled Beam at Constrained Torsion by Boundary Element Method. J. Theor. Appl. Mechanics, 42 (2012), No. 2, 43-54.

[7] Man-B \& W. Vibration Characteristics of Two-stroke Low Speed Diesel Engines, P.8708-165.

[8] Man-B \& W. An Introduction to Vibration Aspects of Two-stroke Diesel Engines in Ships, P. 8809-190.

[9] Bryndum, L., B. Jakobsen, C. Jensen. Vibration Aspects of Long-Stroke Diesel Engines, 2nd Int. Marine Eng. Conf., Shanhai, 1992.

[10] Marinov, B., AL. B. Kazakoff, P. Raykov. Modelling of Big Engines Frequency and Dynamic Behaviour, 12th Nat. Cong. Theor. Appl. Mechanics, 23-26 September 2013, Bulgaria, Varna, Sts Constantine and Helena, 2013.

[11] Marinov, B., Al. B. Kazakoff, P. Raykov. Modelling of Engines Dynamic Behaviour for Marine Vessels. Compt. rend. De l'Acad. Bulgare des Sciences, 67 (2014), No. 3, 419-426.

[12] Marinov, B., Al. B. Kazakoff, M. Tsveov. Frequency and Modal Analysis of a Five Mass Power Transmission Line of a Ship. Compt.rend. De l'Acad. Bulgare des Sciences, 69 (2016), No. 1, 75-84.

[13] Beer, F. P., E. R. Johnston, Jr. J. T. Dewolf, D. F. Mazurek. Mechanics of Materials, USA, McGrow Hill, Fifth Edition, 2009.

[14] Hearn, E. J. Mechanics of Materials 1, UK, Oxford, Jordan Hill, Linacre House, Third Edition, 1997.

[15] Hearn, E. J. Mechanics of Materials 2, UK, Oxford, Jordan Hill, Linacre House, Third Edition, 1997.

[16] Milkov, V. D. Strength of Materials, Bulgaria, Varna, Technical University, 2008 (in Bulgarian). 